\title{
Risk Factors Associated With Early Recurrence of Borderline Resectable Pancreatic Ductal Adenocarcinoma After Neoadjuvant Chemoradiation Therapy and Curative Resection
}

\author{
NOBUHIRO TSUCHIYA, RYUSEI MATSUYAMA, TAKASHI MURAKAMI, \\ YASUHIRO YABUSHITA, YU SAWADA, TAKAFUMI KUMAMOTO and ITARU ENDO \\ Department of Gastroenterological Surgery, Graduate School of Medicine, \\ Yokohama City University, Yokohama, Japan
}

\begin{abstract}
Background/Aim: To identify risk factors of early recurrence after neoadjuvant chemoradiation therapy (NACRT) and curative pancreatectomy in patients with borderline resectable (BR) pancreatic ductal adenocarcinoma (PDAC). Patients and Methods: Sixty-one patients with BR-PDAC who underwent curative resection after NACRT during July 2009June 2014 were included. Patients were divided into early recurrence (i.e., developed recurrence within 1 year after pancreatectomy; $n=30$ ) and late/non-recurrence groups $(n=31)$. The patient characteristics, clinicopathological factors of early recurrence, and survival time were retrospectively compared between groups. Results: In the univariate analysis, the maximum standardized uptake value (SUVmax), microvascular invasion, and lymph node metastasis were associated with early recurrence. In the multivariate analysis, the pre-NACRT SUVmax and microvascular invasion in the early recurrence group were significantly different from that in the late/nonrecurrence group. A pre-NACRT SUVmax $>4.1$ was an independent predictor of poor recurrence-free and overall survival. Conclusion: SUVmax and microvascular invasion are independent predictors of poor recurrence-free and overall survival after NACRT for BR-PDAC. Although complete pancreatectomy after NACRT was performed, approximately half of the patients had recurrence within 1 year.
\end{abstract}

Despite significant advances in cancer diagnosis and treatment, pancreatic ductal adenocarcinoma (PDAC) still

Correspondence to: Itaru Endo, Department of Gastroenterological Surgery, Graduate School of Medicine, Yokohama-city University, 3-9 Fukuura, Kanazawa-ku, Yokohama 236-0004, Japan. Tel: +81 457872650, Fax: +81 457829161, e-mail: endoit@yokohamacu.ac.jp

Key Words: Pancreatectomy, pancreatic ductal carcinoma, positronemission tomography, recurrence, risk factors. has a very poor prognosis. It is estimated that there will be 55,440 new cases and 44,330 deaths attributable to pancreatic cancer by 2018; approximately, $96 \%$ of these will be PDAC (1). Although margin-negative complete surgical resection remains the only treatment with curative intent with a 5-year survival rate of $20-25 \%$, only $15 \%$ to $20 \%$ of patients have resectable disease at the time of diagnosis (2). Several advances in the management of PDAC have recently been reported. For instance, based on an expert consensus report, PDAC can be broadly categorized as resectable, borderline resectable (BR), and unresectable based on tumor encasement of arterial (celiac axis, superior mesenteric artery [SMA], and common hepatic artery [CHA]), or venous (superior mesenteric vein [SMV] and portal vein) structures (3). These definitions have facilitated a more uniform anatomical classification of PDAC and reliable cross-study comparisons.

BR-PDAC is frequently associated with positive surgical margins and poor prognosis after resection compared to resectable-PDAC because frequent positive surgical margins are expected with upfront resection (4). Therefore, neoadjuvant chemoradiation therapy (NACRT) strategies are increasingly being employed for $\operatorname{BR}-\operatorname{PDAC}(5,6)$. These strategies include early initiation of systemic therapy in these patients in contrast to a surgery-first approach where up to half of the patients may not receive adjuvant therapy due to postoperative complications or a decline in functional status. Theoretically, the neoadjuvant approach downstages nodal disease, increases the rate of margin negative resection, and helps identify patients at risk of early disease progression. Several researchers have reported favorable outcomes with NACRT followed by complete surgical resection for BRPDAC compared to patients who did not undergo surgery (6, 7). However, $60 \%$ of patients experience local and systemic recurrence within the first year after curative surgery and survival outcomes are negatively influenced by early recurrence following pancreatic resection (8). Additionally, 
few studies have described the risk factors associated with early recurrence in patients with BR-PDAC after NACRT. Therefore, identification of prognostic factors associated with early recurrence in patients with BR-PDAC after NACRT is of paramount importance in order to improve patient outcomes. The present study aimed to identify risk factors of early recurrence and characterize treatment and outcomes of patients with BR-PDAC after NACRT and surgical resection.

\section{Materials and Methods}

Patients. The medical records of patients with BR-PDAC who underwent NACRT between July 2009 and June 2014 at the Department of Gastroenterological Surgery, Yokohama City University Hospital, Japan, were retrospectively reviewed. Clinical staging was determined by thin slice contrast enhanced computed tomography (CT), magnetic resonance imaging, endoscopic retrograde cholangiopancreatography, endoscopic ultrasound, and $\left[{ }^{18} \mathrm{~F}\right]$-2-deoxy-D-glucose positron emission tomography/CT $\left({ }^{18} \mathrm{~F}\right.$ FDG-PET/CT). According to the National Comprehensive Cancer Network (NCCN) Clinical Practice Guidelines 2009, BR-PDAC has been defined as "involvement of $<180$ degrees of the celiac trunk or SMA, involving only a short segment of the hepatic artery, and occluding only a short segment of the SMV, portal vein, or confluence"(3). Onodera's prognostic nutritional index (PNI) value was calculated using the following formula: 10xserum albumin $(\mathrm{g} / \mathrm{dl})+0.005 \times$ total lymphocyte count $\left(\right.$ per $\left.\mathrm{mm}^{3}\right)$. Modified Glasgow Prognostic Score (mGPS) was constructed as follows: patients with elevated CRP $(>1.0 \mathrm{mg} / \mathrm{dl})$ and decreased serum albumin $(<3.5 \mathrm{~g} / \mathrm{dl})$ levels were assigned a score of 2 . Patients with elevated CRP $(>1.0$ $\mathrm{mg} / \mathrm{dl})$ and albumin $(\geq 3.5 \mathrm{~g} / \mathrm{dl})$ levels were assigned a score of 1 and those with a normal CRP level $(\leq 1.0 \mathrm{mg} / \mathrm{dl})$ were assigned a score of 0 .

This study was approved by the Ethical Review Board Committee (B 131107020) of Yokohama City University Hospital, Yokohama, Japan. Surgery was performed after all possible alternative procedures or treatments had been explained to the patients and they had provided their informed consent. The treatments were performed in accordance with the ethical standards described by the Declaration of Helsinki.

Procedures. In this study, the dosage of tegafur/gimeracil/oteracil (S-1) administered for NACRT was based on the results of a phase II study of gemcitabine $+\mathrm{S}-1$ in which $1000 \mathrm{mg} / \mathrm{m}^{2}$ of gemcitabine was combined with a daily dose of $60 \mathrm{mg} / \mathrm{m}^{2}$ of S-1 (9). Patients received 3 cycles of gemcitabine intravenously, on days 8 and 15 of a 21-day cycle, and oral S-1, twice daily on days 1-14. Radiotherapy was administered concurrently with S-1 daily for 14 days; a total dose of 30 Gy in 10 fractions. Following NACRT, patients had their disease re-staged by thin slice contrast CT and ${ }^{18}$ F-FDG-PET/CT. Patients were examined by our multidisciplinary pancreatic cancer treatment team. If locoregional disease was stable or there was less or no distant metastatic disease, the patients underwent pancreatectomy with standard lymph node dissection within 6 weeks of NACRT. The perineural tissue around the major arteries was dissected by half of the circumference to clear the margin. All patients who underwent resection received gemcitabine or S-1 treatment for 6 months. If tumor progression was apparent after NACRT, the need for additional chemotherapy or chemoradiotherapy was determined on an individual basis. Pathology and operative reports were reviewed to evaluate the margin status and details about the resection. Curative resection was defined as resection with a tumor-free margin and no residual tumors in other organs (R0). Resection margins were defined as positive (R1) if malignant cells were found within $1 \mathrm{~mm}$ of the pancreatic resection margin, the plexus around SMA or CHA, bile duct, duodenum, or retroperitoneal tissue. If vein resection was performed, the vein margin was examined by the pathologist. The histologic grade of NACRT response was evaluated by the Evans grading system (I, 0-10\% tumor cells destroyed; IIa, 10-50\% of tumor cells destroyed; IIb, 50-90\% of tumor cells destroyed; III, $90 \%$ of tumor cells destroyed; and IV, no viable tumor cells) (10).

Early recurrence was defined as recurrence within 1 year after operation. The early recurrence patients were distinguished from the late/non-recurrence patients, which included patients who developed recurrence more than 1 year after resection, and patients who did not develop recurrence. The medical records of all patients were reviewed retrospectively and follow-up data for contrast CT were evaluated every 3 months. The laboratory results, including tumor markers, tumor pathology, and recurrence, were collected for each patient. The first site of disease recurrence was defined as follows: locoregional recurrence, development of a new low-density mass in the region of the pancreatic bed and root of the mesentery; distant metastasis, new low-density region in the liver or lung; and peritoneal dissemination, new ascites on $\mathrm{CT}$ and subsequently confirmed by cytological examination. Recurrence-free survival (RFS) was calculated as the time from the date of surgery to the date of initial recurrence. Overall survival (OS) was calculated as the time from the date of initial treatment to the date of death. The length of the tumor was estimated based on the contrast CT image before treatment and on the resected specimen.

Statistics. Continuous variables were expressed as means with standard deviations and compared using the independent Student's $t$-test. Categorical variables were expressed as numbers and percentages and compared using the Chi-squared or Fisher's exact test. A receiver operating characteristics (ROC) curve was constructed to determine the optimal cutoff value of tumor markers and median maximum standardized uptake value (SUVmax). ROC curve analyses were conducted for RFS. Univariate and multivariate logistic regression models were used to determine factors associated with early recurrence following initial surgery. Relative risks were expressed as hazard ratios (HR) and 95\% confidence intervals (CI). Variables with a $p$-value $<0.1$ by univariate analysis were included in the multivariate model. Two-tailed $p$-values $<0.05$ were considered statistically significant. Statistical analyses were performed using SPSS 22.0 (Chicago, IL, USA).

\section{Results}

Patient characteristics. Of the 85 patients with BR-PDAC who underwent NACRT, 84 patients completed NACRT (Figure 1). The median follow-up times for all patients were 19.0 months (range=3.2-85.5 months). Approximately, $24.7 \%(21 / 85)$ of patients did not undergo pancreatectomy because they had progressive disease (PD) or intraoperative 


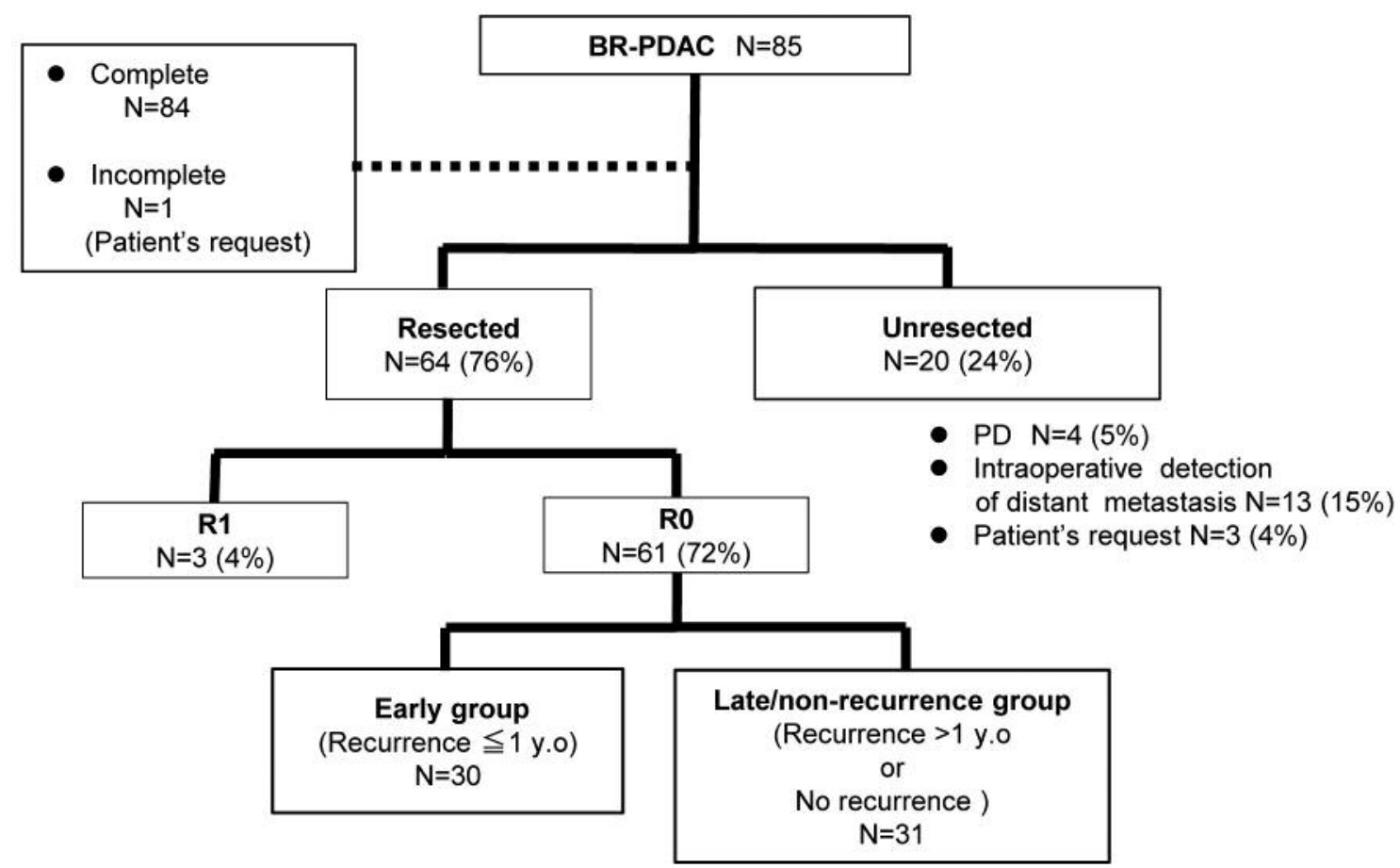

Figure 1. Flow-chart of the selection of patients included in the analysis.

A

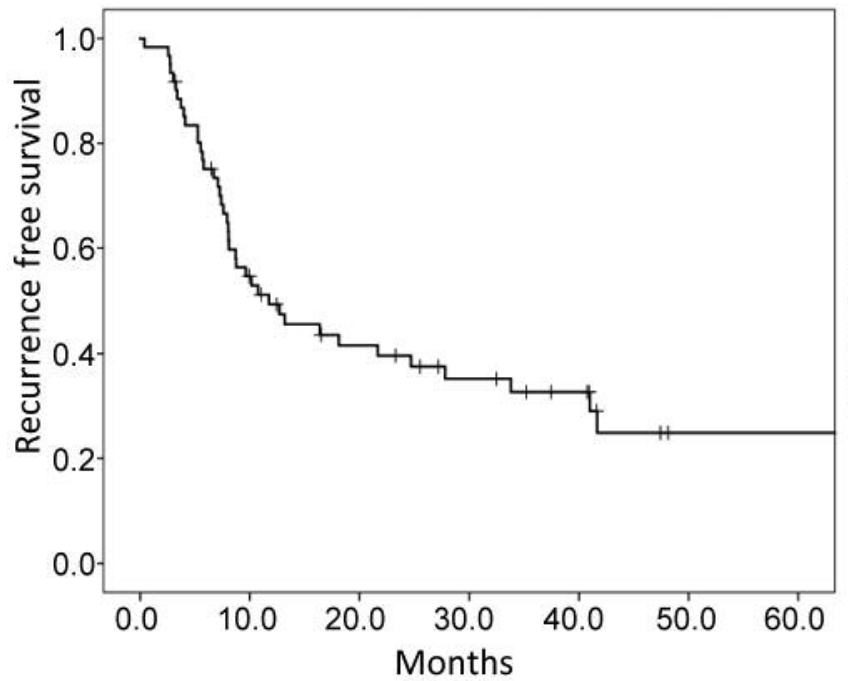

B

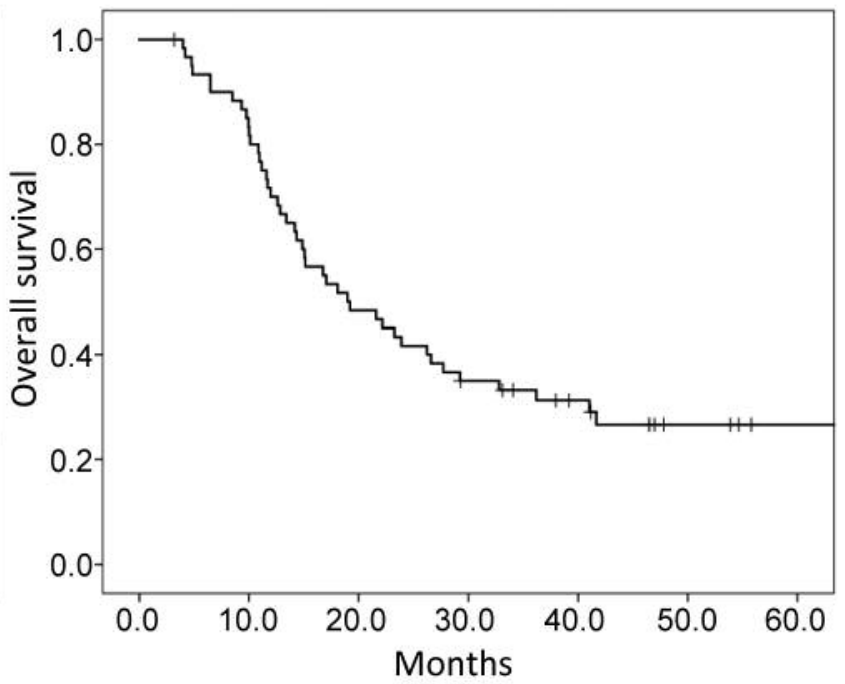

Figure 2. Kaplan-Meier curves for recurrence-free survival (A) and overall survival (B). The median recurrence-free survival and overall survival of all patients were 12 months and 19 months, respectively.

detection of distant metastasis, or that was the patient's request. The adverse events related to NACRT did not prevent any of the patients from completing treatment or lead to any subsequent surgical morbidity. There were no cases of liver or renal failure and there were no deaths related to NACRT or surgery. Approximately 76\% (64/85) of patients underwent pancreatectomy, and the R0 resection rate was $95.3 \%$ (61/64). All patients who were admitted for surgery 
Table I. Patient characteristics.

\begin{tabular}{|c|c|c|c|}
\hline & Early group $n=30$ & Late/non-recurrence groupn=31 & $p$-Value \\
\hline Age & $63.8 \pm 9.08$ & $67.2 \pm 7.94$ & 0.122 \\
\hline \multicolumn{4}{|l|}{ Gender } \\
\hline Male & $17(46.7 \%)$ & $18(58.1 \%)$ & \multirow[t]{2}{*}{0.912} \\
\hline Female & $13(52.3 \%)$ & $13(41.9 \%)$ & \\
\hline \multicolumn{4}{|l|}{ NCCN resectability } \\
\hline BR-PV/SMV & $24(80.0 \%)$ & $22(87.1 \%)$ & \multirow[t]{2}{*}{0.413} \\
\hline BR-A & $6(20.0 \%)$ & $9(22.9 \%)$ & \\
\hline PNI & $47.3 \pm 5.9$ & $48.4 \pm 5.3$ & 0.438 \\
\hline NLR & $2.4 \pm 0.8$ & $2.7 \pm 1.4$ & 0.198 \\
\hline $\mathrm{mGPS} \geq 1$ & $9(30.0 \%)$ & $9(22.9 \%)$ & 0.934 \\
\hline \multicolumn{4}{|l|}{ Dose intensity (\%) } \\
\hline GEM & $85.0 \pm 16.7$ & $85.0 \pm 22.5$ & 0.990 \\
\hline S1 & $94.4 \pm 17.3$ & $94.4 \pm 20.9$ & 0.995 \\
\hline Adverse event $(\geq \mathrm{G} 3)$ & $20(66.7 \%)$ & $17(54.8 \%)$ & 0.490 \\
\hline Pre-NACRT CEA & $9.1 \pm 17.9$ & $6.0 \pm 7.0$ & 0.374 \\
\hline Post-NACRT CEA & $6.0 \pm 6.9$ & $4.9 \pm 3.5$ & 0.449 \\
\hline Post-resection CEA & $5.8 \pm 10.2$ & $3.7 \pm 2.0$ & 0.269 \\
\hline Pre-NACRT CA19-9 & $533 \pm 1338$ & $1404 \pm 7049$ & 0.509 \\
\hline Post-NACRT CA19-9 & $277 \pm 906$ & $58 \pm 105$ & 0.199 \\
\hline Post-resection CA19-9 & $40.9 \pm 68.5$ & $20.8 \pm 30.1$ & 0.154 \\
\hline Pre-NACRT PET SUVmax & $6.84 \pm 3.32$ & $5.14 \pm 2.96$ & 0.039 \\
\hline Post-NACRT PET SUVmax & $5.01 \pm 1.41$ & $3.97 \pm 1.91$ & 0.021 \\
\hline
\end{tabular}

NCCN: National Comprehensive Cancer Network; BR: borderline resectable; PV: portal vein; SMV: superior mesenteric vein; PNI: Onodera's prognostic nutritional index; NLR: neutrophil/lymphocyte ratio; PLR: platelet/lymphocyte ratio; mGPS, modified Glasgow Prognostic Score; GEM: gemcitabine; S-1: tegafur/gimeracil/oteracil; NACRT: neoadjuvant chemoradiation therapy; PET: positron emission tomography/computed tomography; SUVmax: median maximum standardized uptake value.

were discharged home. During follow-up, early recurrence was noted in $30(49.2 \%)$ patients. Of the remaining patients, $7(11.5 \%)$ had late recurrence and $24(39.3 \%)$ patients did not have any recurrence. There were $35(57.4 \%)$ patients who died of disease progression, 2 (3.3\%) patients who were alive with disease; and $16(26.2 \%)$ patients who were alive with no evidence of disease at the end of the study. The median OS was 19 months, and the cumulative incidence of recurrence at 1,3 , and 5 years was $50.7 \%, 60.4 \%$, and $75.1 \%$, respectively (Figure $2 \mathrm{~A}$ and $\mathrm{B}$ ). Furthermore, the mortality rate of patients who were recognized as having both an SUVmax $>4.1$ and microvascular invasion was significantly higher than that of patients without these 2 factors $(91.3 \%$ vs. $30.0 \% ; p=0.01)$.

Risk factors associated with early recurrence. Regarding preoperative risk factors, there were no significant differences in NCCN resectability, dose intensity, and tumor markers, including carbohydrate antigen 19-9 (CA19-9), between the 2 groups (Table I). Early recurrence occurred more commonly in patients with a significantly higher preand post-NACRT SUVmax than those who were recurrencefree for 1 year after curative pancreatectomy followed by NACRT (6.84 vs. 5.14, $p=0.039$ and 5.01 vs. 3.97, $p=0.021$, respectively). However, the reduction in the SUVmax was not different between the early and late recurrence groups. Having microvascular invasion $(p=0.003)$, portal vein invasion $(p=0.043)$, and positive lymph nodes $(p=0.015)$ were significant early recurrence risk factors (Table II). Patients who were recurrence-free for 1 year were likely to benefit more from NACRT, as evaluated by the Evans grading system, than those who had early recurrence. The adjuvant chemotherapy induction rates were almost similar in both groups $(73.3 \%$ and $80.6 \%$ of early and late/nonrecurrence patients, respectively; Table III). The distribution of all anatomic sites and locations of tumor recurrence in the early recurrence group were as follows: liver $(43.3 \%)$, peritoneal carcinomatosis $(20 \%)$, lungs $(20 \%)$, locoregional $(10 \%)$, and lymph nodes $(6.7 \%)$. Liver metastasis was likely to occur earlier than metastasis at other sites (Table IV). Based on the ROC curves for RFS for 1 year, with an area under the curve of 0.662 , we identified an SUVmax of 4.1 pre-NACRT as the optimal cutoff value (sensibility, $86.7 \%$; specificity, $43.3 \%$ ). On multivariate analysis, pre-NACRT SUVmax $>4.1 \quad(\mathrm{HR}=2.8 ; 95 \% \mathrm{CI}=1.2-6.4 ; p=0.017)$ and microvascular invasion $(\mathrm{HR}=3.0 ; 95 \% \mathrm{CI}=1.5-5.8 ; p=0.002)$ were independent risk factors associated with early recurrence following curative pancreatectomy after NACRT 
Table II. Histological outcome.

\begin{tabular}{lccc}
\hline & Early group n=30 & Late/non-recurrence groupn=31 & $p$-Value \\
\hline Size (cm) & $3.27 \pm 1.09$ & $3.07 \pm 1.69$ & 0.588 \\
Grade (poor differentiated) & $8(26.7 \%)$ & $11(35.5 \%)$ & 0.457 \\
Lymphatic invasion & $7(23.3 \%)$ & $6(19.4 \%)$ & 0.704 \\
Vascular invasion & $20(66.7 \%)$ & $9(29.0 \%)$ & 0.003 \\
Perineural invasion & $28(93.3 \%)$ & $26(83.9 \%)$ & 0.246 \\
Portal vein invasion & $13(43.3 \%)$ & $6(19.4 \%)$ & 0.043 \\
Arterial invasion & $3(10.0 \%)$ & $0(0 \%)$ & 0.071 \\
Nodal status & $19(63.3 \%)$ & $10(32.3 \%)$ & 0.015 \\
Positive & $1.32 \pm 1.85$ & $0.68 \pm 1.42$ & 0.137 \\
Positive nodes & $18.7 \pm 11.9$ & $20.4 \pm 9.6$ & 0.537 \\
Harvested nodes & & & 0.232 \\
Stage & $10(33.3 \%)$ & $15(48.4 \%)$ & 0.072 \\
I/II/III & $20(66.7 \%)$ & $16(51.6 \%)$ & \\
IVa/IVb & & & \\
Evans classification & $4(13.3 \%)$ & $2(6.5 \%)$ & \\
I & $26(86.7 \%)$ & $6(19.3 \%)$ & $0(0 \%)$ \\
IIa/IIb & $0(0 \%)$ & $0(0 \%)$ & \\
III & & & \\
IV & & & \\
\hline
\end{tabular}

Table III. Adjuvant chemotherapy

\begin{tabular}{lccc}
\hline & Early group $\mathrm{n}=30$ & Late/non-recurrence groupn=31 & $p$-Value \\
\hline Induction rate & $22(73.3 \%)$ & $25(80.6 \%)$ & 0.497 \\
Regimens & $4(13.3 \%)$ & $0(0 \%)$ & $13(41.9 \%)$ \\
GEM & $9(30.0 \%)$ & $12(38.7 \%)$ & $78.8 \pm 46.5$ \\
S1 & $9(30.0 \%)$ & 0.513 \\
GEM+S1 & $71.1 \pm 30.1$ & \\
Postoperative day of initiation &
\end{tabular}

Table IV. Recurrence pattern.

\begin{tabular}{lcc}
\hline & Early group n=30 & Late/non-recurrence groupn=31 \\
\hline Site of recurrence & & \\
$\quad$ Liver & $13(43.3 \%)$ & $1(3.2 \%)$ \\
Peritoneal & $6(20.0 \%)$ & $2(6.5 \%)$ \\
Local & $3(10.0 \%)$ & $2(6.5 \%)$ \\
Lymph node & $2(6.7 \%)$ & $2(6.5 \%)$ \\
Lung & $6(20.0 \%)$ & $4(13.3 \%)$ \\
Other & $1(3.3 \%)$ & $2(6.5 \%)$ \\
\hline
\end{tabular}

for BR-PDAC (Table V). Furthermore, in the KaplanMeier analysis, having an SUVmax $>4.1$ (Figure 3A, B; RFS, $8 v s .41$ months, $p=0.004$ and OS, 15 months $v s$. not reached, $p=0.006$ ) and microvascular invasion (Figure $3 \mathrm{C}$, D; RFS, 8 months $v s$. not reached, $p<0.001$ and OS, $15 v s$. 33 months, $p=0.011$ ) were significant factors for poor RFS and OS.

\section{Discussion}

Although surgical resection of BR-PDAC represents a potentially curative treatment, the high recurrence rate after pancreatectomy results in a poor prognosis (4-6). Therefore, understanding and predicting recurrence is important for improving the prognosis of BR-PDAC. Based on our findings, 
Table V. Univariate and multivariate analyses of predictors of RFS

\begin{tabular}{|c|c|c|c|c|}
\hline \multirow[t]{2}{*}{ Parameters } & \multicolumn{2}{|c|}{ Univariate analysis } & \multicolumn{2}{|c|}{ Multivariable analysis } \\
\hline & $\mathrm{HR}(95 \% \mathrm{CI})$ & $p$-Value & $\mathrm{HR}(95 \% \mathrm{CI})$ & $p$-Value \\
\hline Age ( $>60$ years) & $0.6(0.3-1.3)$ & 0.187 & & \\
\hline Gender (male) & $1.0(0.5-2.0)$ & 0.935 & & \\
\hline \multicolumn{5}{|l|}{ NCCN resectability } \\
\hline BR-PV/SMV & $1.0(0.3-1.3)$ & 0.976 & & \\
\hline BR-A & $1.0(0.4-2.7)$ & 0.970 & & \\
\hline PNI $(<40)$ & $1.4(0.5-4.0)$ & 0.502 & & \\
\hline mGPS $(\geq 1)$ & $1.0(0.5-2.0)$ & 0.978 & & \\
\hline Dose intensity $(<80)$ & $0.8(0.4-1.6)$ & 0.488 & & \\
\hline Adverse event $(\geq \mathrm{G} 3$ ) & $1.5(0.6-3.3)$ & 0.355 & & \\
\hline Pre-NACRT CEA $(>5 \mathrm{U} / \mathrm{ml})$ & $1.0(0.5-2.1)$ & 0.948 & & \\
\hline Post-NACRT CEA (>5 U/ml) & $0.8(0.4-1.8)$ & 0.612 & & \\
\hline Post-resection CEA (>3.8 U/m) & $0.8(0.4-1.7)$ & 0.525 & & \\
\hline Pre-NACRT CA19-9 (>200 U/ml) & $1.6(0.8-3.5)$ & 0.216 & & \\
\hline Post-NACRT CA19-9 (>67 U/ml) & $1.9(1.0-3.8)$ & 0.068 & & \\
\hline Post-resection CA19-9 (>37 U/ml) & $1.9(0.8-4.3)$ & 0.125 & & \\
\hline Pre-NACRT PET SUVmax (>4.1) & $3.6(1.3-10.5)$ & 0.016 & $2.8(1.2-6.4)$ & 0.017 \\
\hline Post-NACRT PET SUVmax $(>3.6)$ & $2.9(1.1-7.6)$ & 0.029 & $1.2(0.6-2.5)$ & 0.549 \\
\hline Size $(>3.5 \mathrm{~cm})$ & $1.5(0.7-3.1)$ & 0.313 & & \\
\hline Grade (poor differentiated) & $0.9(0.4-1.7)$ & 0.664 & & \\
\hline Lymphatic invasion & $1.0(0.4-2.4)$ & 0.955 & & \\
\hline Vascular invasion & $2.9(1.3-6.2)$ & 0.007 & $3.0(1.5-5.8)$ & 0.002 \\
\hline Perineural invasion & $1.6(0.8-3.3)$ & 0.225 & & \\
\hline Portal vein invasion & $2.0(0.9-4.1)$ & 0.069 & & \\
\hline Artery invasion & $2.7(0.8-9.1)$ & 0.101 & & \\
\hline Nodal metastasis & $2.3(1.1-4.9)$ & 0.028 & $1.6(0.7-3.6)$ & 0.239 \\
\hline stage $(>\mathrm{III})$ & $1.6(0.8-3.5)$ & 0.207 & & \\
\hline Evans classification (>grade IIa) & $1.6(0.8-3.4)$ & 0.193 & & \\
\hline Adjuvant chemotherapy & $0.6(0.2-1.3)$ & 0.156 & & \\
\hline
\end{tabular}

NCCN: National Comprehensive Cancer Network; BR: borderline resectable; PV: portal vein; SMV: superior mesenteric vein; PNI: Onodera's prognostic nutritional index; NLR: neutrophil/lymphocyte ratio; PLR: platelet/lymphocyte ratio; mGPS: modified Glasgow Prognostic Score; NACRT: neoadjuvant chemoradiation therapy; PET: positron emission tomography/computed tomography; SUVmax: median maximum standardized uptake value.

the ${ }^{18}$ F-FDG-PET/CT SUVmax and microvascular invasion could be utilized in predicting early tumor recurrence for BRPDAC patients after NACRT. The potential role of FDG uptake values in the prediction of prognosis has been recently reported in several meta-analyses. High SUV values at diagnosis are more highly associated with poor survival than low SUV values in a variety of cancers, such as head and neck cancer, hepatocellular carcinoma, and bone and soft tissue sarcoma (11-13). To date, ${ }^{18}$ F-FDG-PET/CT has been considered the imaging modality that is most commonly used for diagnosis, staging, evaluating response to treatment, and detecting postoperative recurrence and metastasis in PDAC. Furthermore, some researchers have also reported the prognostic value of preoperative ${ }^{18} \mathrm{~F}$-FDG-PET/CT patients with SUVmax cutoff values of 3.3-7.0 after resection, including locally advanced PDAC (14-16). Other ${ }^{18}$ F-FDGPET/CT parameters, such as metabolic tumor volume and total lesion glycolysis, which reflect both metabolic activity and tumor volume, have been shown to be independent preoperative risk factors for OS and RFS (16). However, the predictive value of ${ }^{18} \mathrm{~F}$-FDG-PET/CT in patients with $\mathrm{BR}$ PDAC after NACRT has not been previously studied. In evaluating the predictive value of SUVmax in these patients, we found that a cutoff value of 4.1 was significantly associated with 1-year recurrence and survival rates after tumor recurrence, with a SUVmax $>4.1$ being a predictor of poor survival after recurrence. These findings suggest that ${ }^{18} \mathrm{~F}-\mathrm{FDG}-$ $\mathrm{PET} / \mathrm{CT}$ might be a tool to help determine which patients require more aggressive neoadjuvant therapy before surgery.

Although an elevation of preoperative or postoperative tumor markers has been reported to be a predictor of poor survival, there were no significant preoperative and postoperative differences in tumor marker levels, including CA19-9, between the early recurrence and late/non-recurrence groups in our study (17-19). Nevertheless, a significant reduction in CA19-9 was evident after NACRT, and there was 
A

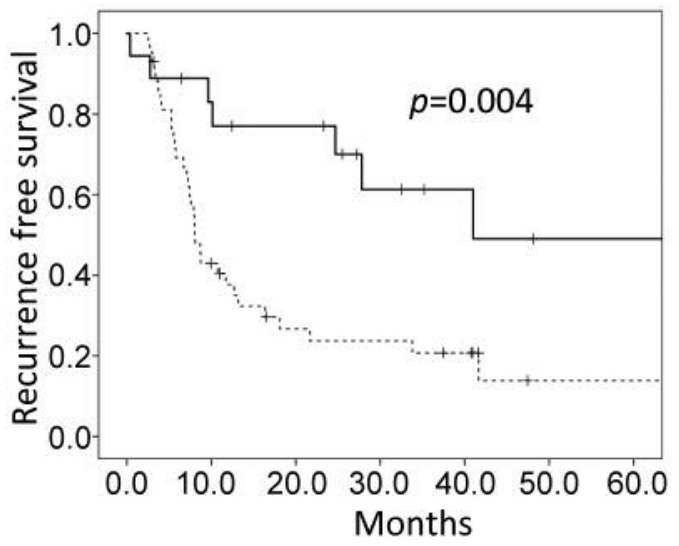

B

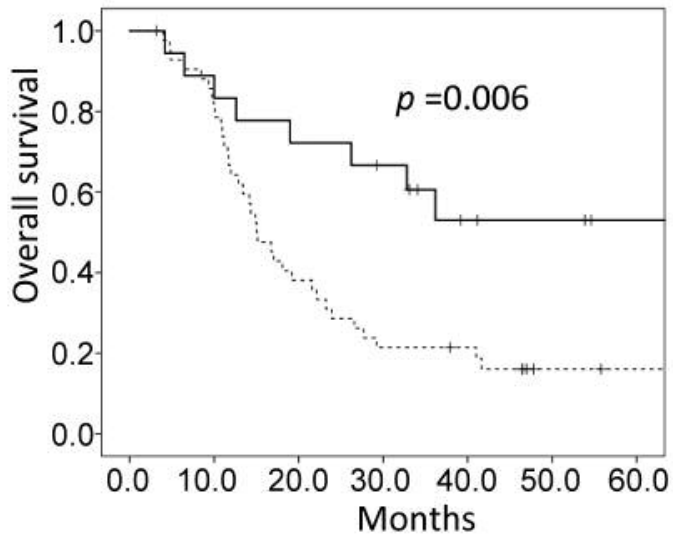

$\begin{array}{ll}- & \text { pre PET SUVmax }(\leq 4.1) \\ - & \text { pre PET SUVmax }(>4.1)\end{array}$

C

D

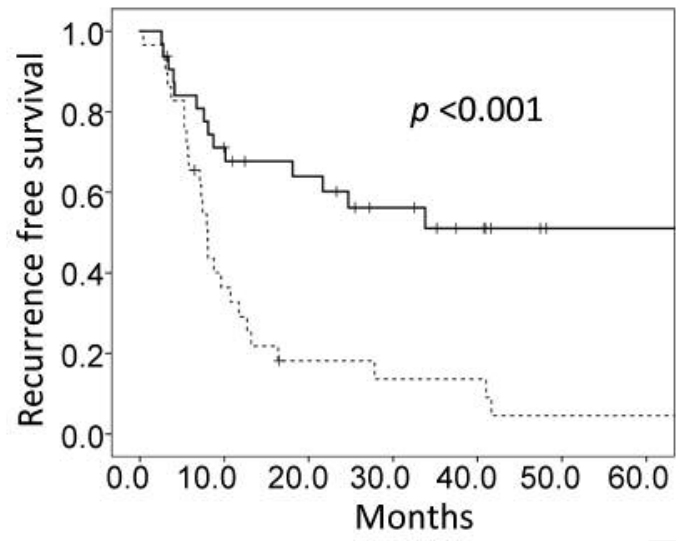

二- $\quad \begin{aligned} & \mathrm{v} \\ & \mathrm{v}+\end{aligned}$

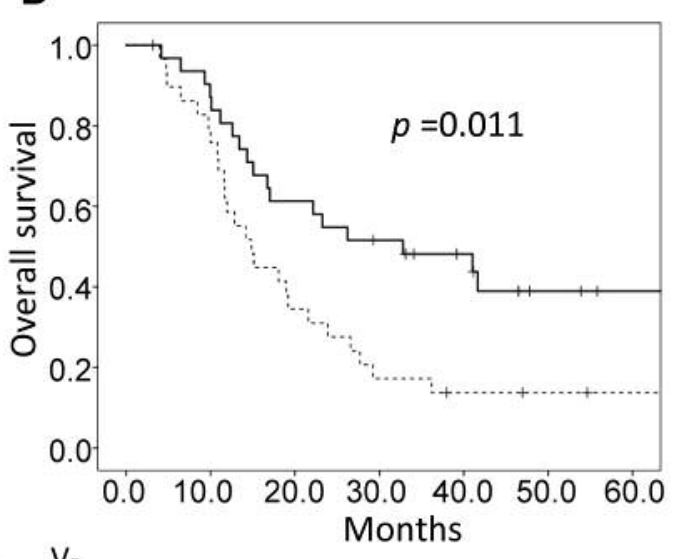

Figure 3. Kaplan-Meier curves for recurrence-free survival (RFS) and overall survival (OS) based on the maximum standard uptake value (SUVmax) and microvascular invasion. Patients with an SUVmax $>4.1$ had a significantly poorer median RFS and OS compared to those with an SUVmax $\leq 4.1$ ( $A$ and B; RFS, 8 vs. 41 months, $p=0.004$; OS, 15 months vs. not reached, p=0.006). Patients with microvascular invasion (v+) had a significantly poorer median RFS and OS compared to those with no microvascular invasion ( $v$-) (C and D; RFS, 8 months vs. not reached, $<<0.001$; OS, 15 vs. 33 months, $p=0.011$ ).

no improvement in the disease stage. Moreover, upon evaluation of the effect of NACRT according to the Evans grading system, the percentage of patients with a grade $>$ IIa was $90.2 \%$ after NACRT (Evans I, 6 patients; IIa, 25 patients; IIb, 24 patients; and III, 6 patients), which is consistent with several studies that reported an NACRT response rate of approximately $89.4 \%$ (20). Although the patients in the earlyrecurrence group were likely to have fewer anticancer effects of NACRT than those in the late/non-recurrence group, as evaluated by the Evans grading system, there was no difference in the reduction of CA19-9 levels between these 2 groups. Therefore, there is a discrepancy between the significant reduction in CA19-9 levels and tumor regression rates.
Currently, the standard of care for BR-PDAC is NACRT followed by surgery. NACRT results in greater survival and time to local recurrence compared with upfront resection. Moreover, there was a lower rate of lymphovascular invasion and smaller tumor size in these patients. Given the potential benefits, NACRT can be useful in patients with unfavorable prognostic features during the preoperative stage. However, PDAC maintains a poor prognosis due to an early appearance of metastases (21). These outcomes support a systemic approach of PDAC from an early stage. Therefore, accurate preoperative diagnosis and staging for PDAC are crucial not only to detect local invasion and distant metastases when assessing resectability, but also to select 
patients who might benefit more from NACRT instead of upfront surgery (i.e., those with more aggressive tumor biology and at higher risk of early recurrence after surgery) (21).

While the main goal of any neoadjuvant strategy is to prolong survival, another goal is to improve $\mathrm{R} 0$ resection because positive resection margins have been shown to be associated with poor survival (22) (23). Based on previous trials, the $\mathrm{R} 1$ resection rate in adjuvant therapy has been reported as $24-42 \%(24,25)$, whereas the margin positive rate in neoadjuvant therapy has been $<10 \%$ in selected centers (5). In the current study, $>90 \%$ of patients had R0 resection after NACRT, which suggests that neoadjuvant therapy increases the likelihood of R0 resection among BRPDAC.

Although R0 resection for BR-PDAC after NACRT was performed, approximately half of the patients had recurrence within 1 year. Liver metastases were the most common type of tumor relapse in these patients. These findings can be influenced by the possible presence of occult metastases at the time of operation, which could be interpreted as early recurrence. Some researchers report that $<58 \%$ of patients with locally advanced PDAC have occult distant metastases at the time of operation $(26,27)$. In this study, almost all patients with early recurrence were diagnosed with distant metastases. Therefore, there is a chance that some of these "recurrences" could have already been present at the time of surgery. Additionally, an increased R0 resection rate and local control in advanced PDAC patients did not translate into significantly improved survival in recent meta-analyses (28). The MD Anderson Cancer Center group found that $45 \%$ of patients had recurrence in distant organs such as lung, liver, or bone (6), indicating the need for effective systemic therapy. These results and our current findings suggest that in addition to improved R0 rates, effective systemic neoadjuvant and adjuvant treatment is also important for improving survival. In this sense, it is possible that newer neoadjuvant chemotherapeutic regimens (gemcitabine-nab-paclitaxel and FOLFIRINOX), which are more potent than gemcitabine $+\mathrm{S}-1$, could improve overall oncologic outcomes. In fact, several phase II clinical trials have been initiated to evaluate the potential efficacy of neoadjuvant treatment with gemcitabine-nab-paclitaxel and FOLFIRINOX in BR-PDAC patients $(29,30)$. No improvement in RFS with adjuvant therapy was detected in BR-PDAC patients after NACRT. This could be the result of administration of S-1 to almost all patients who received adjuvant therapy, which may have reduced the efficacy of this treatment. Additionally, predictive markers may be useful when deciding which BR-PDAC patients may benefit from adjuvant chemotherapy following complete resection and which chemotherapeutic agents may be used in individual patients to maximize survival. Both SUVmax $>4.1$ and microvascular invasion were strongly correlated with poor prognosis in this study. Therefore, a more powerful regimen, such as gemcitabine-nab-paclitaxel and modified FOLFIRINOX, should be provided to these patients to improve their prognosis.

This study has several limitations. Certainly, the retrospective nature of our cohort study and the small sample size limited the possibility of inferring robust conclusions. However, patient selection, diagnostic workup, and surgical treatment were conducted in the same institution by an established multidisciplinary team. Moreover, although ROC curves showed a SUVmax $\geq 4.1$ as the best cutoff value to predict RFS and OS in the present study, no consensus has yet been established in the literature about the optimal SUVmax cutoff (15). This is a reasonable concern for the use of ${ }^{18} \mathrm{~F}-\mathrm{FDG}-\mathrm{PET} / \mathrm{CT}$, and further investigations in a larger sample size are needed to achieve a standardization of the SUVmax cutoff value.

In conclusion, an SUVmax $>4.1$ and microvascular invasion are independent predictors of poor RFS and OS in patients with BR-PDAC after NACRT. NACRT for BRPDAC can help achieve a clear margin with good local control. Although R0 resection for BR-PC after NACRT was performed, approximately half of the patients had recurrence within 1 year. Therefore, more powerful and effective chemotherapy is needed to reduce distant metastasis, that would improve patient outcomes and reduce healthcare expenses.

\section{Conflicts of Interest}

The Authors declare no conflict of interest regarding this study.

\section{Authors' Contributions}

Nobuhiro Tsuchiya designed the study, and wrote the initial draft of the manuscript with support from Itaru Endo. All other authors have contributed to data collection and approved the final version of the manuscript, and agree to be accountable for all aspects of the work in ensuring that questions related to the accuracy or integrity of any part of the work are appropriately investigated and resolved.

\section{References}

1 Siegel RL, Miller KD and Jemal A: Cancer statistics, 2018. CA Cancer J Clin 68(1): 7-30, 2018. PMID: 29313949. DOI: $10.3322 /$ caac. 21442

2 Oettle H, Post S, Neuhaus P, Gellert K, Langrehr J, Ridwelski K, Schramm H, Fahlke J, Zuelke C, Burkart C, Gutberlet K, Kettner E, Schmalenberg H, Weigang-Koehler K, Bechstein WO, Niedergethmann M, Schmidt-Wolf I, Roll L, Doerken B and Riess H: Adjuvant chemotherapy with gemcitabine vs. observation in patients undergoing curative-intent resection of pancreatic cancer: A randomized controlled trial. JAMA 297(3): 267-277, 2007. PMID: 17227978. DOI: 10.1001/jama.297.3.267 
3 Tempero MA, Malafa MP, Behrman SW, Benson AB, 3rd, Casper ES, Chiorean EG, Chung V, Cohen SJ, Czito B, Engebretson A, Feng M, Hawkins WG, Herman J, Hoffman JP, Ko A, Komanduri S, Koong A, Lowy AM, Ma WW, Merchant NB, Mulvihill SJ, Muscarella P, 2nd, Nakakura EK, Obando J, Pitman MB, Reddy S, Sasson AR, Thayer SP, Weekes CD, Wolff RA, Wolpin BM, Burns JL and Freedman-Cass DA: Pancreatic adenocarcinoma, version 2.2014: Featured updates to the nccn guidelines. J Natl Compr Canc Netw 12(8): 1083-1093, 2014. PMID: 25099441.

4 Abrams RA, Lowy AM, O'Reilly EM, Wolff RA, Picozzi VJ and Pisters PW: Combined modality treatment of resectable and borderline resectable pancreas cancer: Expert consensus statement. Ann Surg Oncol 16(7): 1751-1756, 2009. PMID: 19390900. DOI: 10.1245/s10434-009-0413-9

5 Evans DB, Varadhachary GR, Crane $\mathrm{CH}$, Sun CC, Lee JE, Pisters PW, Vauthey JN, Wang H, Cleary KR, Staerkel GA, Charnsangavej C, Lano EA, Ho L, Lenzi R, Abbruzzese JL and Wolff RA: Preoperative gemcitabine-based chemoradiation for patients with resectable adenocarcinoma of the pancreatic head. J Clin Oncol 26(21): 3496-3502, 2008. PMID: 18640930. DOI: 10.1200/JCO.2007.15.8634

6 Katz MH, Pisters PW, Evans DB, Sun CC, Lee JE, Fleming JB, Vauthey JN, Abdalla EK, Crane CH, Wolff RA, Varadhachary GR and Hwang RF: Borderline resectable pancreatic cancer: The importance of this emerging stage of disease. J Am Coll Surg 206(5): 833-846; discussion 846-838, 2008. PMID: 18471707 DOI: $10.1016 /$ j.jamcollsurg.2007.12.020

7 Takahashi H, Ohigashi H, Gotoh K, Marubashi S, Yamada T, Murata $\mathrm{M}$, Ioka $\mathrm{T}$, Uehara $\mathrm{H}$, Yano $\mathrm{M}$ and Ishikawa $\mathrm{O}$ : Preoperative gemcitabine-based chemoradiation therapy for resectable and borderline resectable pancreatic cancer. Ann Surg 258(6): 1040-1050, 2013. PMID: 23799421. DOI: $10.1097 /$ SLA.0b013e31829b3ce4

8 La Torre M, Nigri G, Lo Conte A, Mazzuca F, Tierno SM, Salaj A, Marchetti P, Ziparo V and Ramacciato G: Is a preoperative assessment of the early recurrence of pancreatic cancer possible after complete surgical resection? Gut Liver 8(1): 102-108, 2014. PMID: 24516708. DOI: 10.5009/ gnl.2014.8.1.102

9 Ueno H, Okusaka T, Furuse J, Yamao K, Funakoshi A, Boku N, Ohkawa S, Yokosuka O, Tanaka K, Moriyasu F, Nakamori S and Sato T: Multicenter phase ii study of gemcitabine and s-1 combination therapy (gs therapy) in patients with metastatic pancreatic cancer. Jpn J Clin Oncol 41(8): 953-958, 2011. PMID: 21715364. DOI: 10.1093/jjco/hyr090

10 Evans DB, Rich TA, Byrd DR, Cleary KR, Connelly JH, Levin B, Charnsangavej C, Fenoglio CJ and Ames FC: Preoperative chemoradiation and pancreaticoduodenectomy for adenocarcinoma of the pancreas. Arch Surg 127(11): 1335-1339, 1992. PMID: 1359851.

11 Xie P, Li M, Zhao H, Sun X, Fu Z and Yu J: 18f-fdg pet or petct to evaluate prognosis for head and neck cancer: A metaanalysis. J Cancer Res Clin Oncol 137(7): 1085-1093, 2011. PMID: 21229262. DOI: 10.1007/s00432-010-0972-y

12 Sun DW, An L, Wei F, Mu L, Shi XJ, Wang CL, Zhao ZW, Li TF and Lv GY: Prognostic significance of parameters from pretreatment (18)f-fdg pet in hepatocellular carcinoma: A metaanalysis. Abdom Radiol (NY) 41(1): 33-41, 2016. PMID: 26830609. DOI: 10.1007/s00261-015-0603-9
$13 \mathrm{Li}$ YJ, Dai YL, Cheng YS, Zhang WB and Tu CQ: Positron emission tomography (18)f-fluorodeoxyglucose uptake and prognosis in patients with bone and soft tissue sarcoma: A metaanalysis. Eur J Surg Oncol 42(8): 1103-1114, 2016. PMID: 27189833. DOI: $10.1016 /$ j.ejso.2016.04.056

14 Maemura K, Takao S, Shinchi H, Noma H, Mataki Y, Kurahara $\mathrm{H}$, Jinnouchi $\mathrm{S}$ and Aikou $\mathrm{T}$ : Role of positron emission tomography in decisions on treatment strategies for pancreatic cancer. J Hepatobiliary Pancreat Surg 13(5): 435-441, 2006. PMID: 17013719. DOI: 10.1007/s00534-006-1102-8

15 Yamamoto T, Sugiura T, Mizuno T, Okamura Y, Aramaki T, Endo $\mathrm{M}$ and Uesaka K: Preoperative fdg-pet predicts early recurrence and a poor prognosis after resection of pancreatic adenocarcinoma. Ann Surg Oncol 22(2): 677-684, 2015. PMID: 25190125. DOI: $10.1245 / \mathrm{s} 10434-014-4046-2$

16 Jung W, Jang JY, Kang MJ, Chang YR, Shin YC, Chang J and Kim SW: The clinical usefulness of $18 \mathrm{f}$-fluorodeoxyglucose positron emission tomography-computed tomography (pet-ct) in follow-up of curatively resected pancreatic cancer patients. HPB (Oxford) 18(1): 57-64, 2016. PMID: 26776852. DOI: 10.1016/ j.hpb.2015.06.001

17 Kondo N, Murakami Y, Uemura K, Hayashidani Y, Sudo T, Hashimoto Y, Nakashima A, Sakabe R, Shigemoto N, Kato Y, Ohge $\mathrm{H}$ and Sueda T: Prognostic impact of perioperative serum ca 19-9 levels in patients with resectable pancreatic cancer. Ann Surg Oncol 17(9): 2321-2329, 2010. PMID: 20336387. DOI: 10.1245/s10434-010-1033-0

18 Abdel-Misih SR, Hatzaras I, Schmidt C, Saab TB, Klemanski D, Muscarella P, Melvin WS, Ellison EC and Bloomston M: Failure of normalization of ca19-9 following resection for pancreatic cancer is tantamount to metastatic disease. Ann Surg Oncol 18(4): 1116-1121, 2011. PMID: 21042945. DOI: 10.1245/ s10434-010-1397-1

19 Motoi F, Murakami Y, Okada KI, Matsumoto I, Uemura K, Satoi S, Sho M, Honda G, Fukumoto T, Yanagimoto H, Kinoshita S, Kurata M, Aoki S, Mizuma M, Yamaue H, Unno M and Multicenter Study Group of Pancreatobiliary S: Sustained elevation of postoperative serum level of carbohydrate antigen 19-9 is high-risk stigmata for primary hepatic recurrence in patients with curatively resected pancreatic adenocarcinoma. World J Surg, 2018. PMID: 30298281. DOI: 10.1007/s00268018-4814-4

20 Nagakawa Y, Hosokawa Y, Nakayama H, Sahara Y, Takishita C, Nakajima T, Hijikata Y, Kasuya K, Katsumata K, Tokuuye K and Tsuchida A: A phase ii trial of neoadjuvant chemoradiotherapy with intensity-modulated radiotherapy combined with gemcitabine and s- 1 for borderline-resectable pancreatic cancer with arterial involvement. Cancer Chemother Pharmacol 79(5): 951-957, 2017. PMID: 28378027. DOI: 10.1007/s00280-017-3288-7

21 Barugola G, Partelli S, Marcucci S, Sartori N, Capelli P, Bassi C, Pederzoli P and Falconi M: Resectable pancreatic cancer: Who really benefits from resection? Ann Surg Oncol 16(12): 33163322, 2009. PMID: 28378027. DOI: 10.1245/s10434-009-0670-7

22 Winter JM, Cameron JL, Campbell KA, Arnold MA, Chang DC, Coleman J, Hodgin MB, Sauter PK, Hruban RH, Riall TS, Schulick RD, Choti MA, Lillemoe KD and Yeo CJ: 1423 pancreaticoduodenectomies for pancreatic cancer: A singleinstitution experience. J Gastrointest Surg 10(9): 1199-1210; discussion 1210-1191, 2006. PMID: 17114007. DOI: 10.1016/ j.gassur.2006.08.018 
23 Konstantinidis IT, Warshaw AL, Allen JN, Blaszkowsky LS, Castillo CF, Deshpande V, Hong TS, Kwak EL, Lauwers GY, Ryan DP, Wargo JA, Lillemoe KD and Ferrone CR: Pancreatic ductal adenocarcinoma: Is there a survival difference for $\mathrm{r} 1$ resections versus locally advanced unresectable tumors? What is a "true" r0 resection? Ann Surg 257(4): 731-736, 2013. PMID: 22968073. DOI: 10.1097/SLA.0b013e318263da2f

24 Regine WF, Winter KA, Abrams RA, Safran H, Hoffman JP, Konski A, Benson AB, Macdonald JS, Kudrimoti MR, Fromm ML, Haddock MG, Schaefer P, Willett CG and Rich TA: Fluorouracil $v s$. gemcitabine chemotherapy before and after fluorouracil-based chemoradiation following resection of pancreatic adenocarcinoma: A randomized controlled trial. JAMA 299(9): 1019-1026, 2008. PMID: 18319412. DOI: 10.1001/jama.299.9.1019

25 Neoptolemos JP, Stocken DD, Bassi C, Ghaneh P, Cunningham D, Goldstein D, Padbury R, Moore MJ, Gallinger S, Mariette C, Wente MN, Izbicki JR, Friess H, Lerch MM, Dervenis C, Olah A, Butturini G, Doi R, Lind PA, Smith D, Valle JW, Palmer DH, Buckels JA, Thompson J, McKay CJ, Rawcliffe CL, Buchler MW and European Study Group for Pancreatic C: Adjuvant chemotherapy with fluorouracil plus folinic acid vs gemcitabine following pancreatic cancer resection: A randomized controlled trial. JAMA 304(10): 1073-1081, 2010. PMID: 20823433. DOI: 10.1001/jama.2010.1275

26 Contreras CM, Stanelle EJ, Mansour J, Hinshaw JL, Rikkers LF, Rettammel R, Mahvi DM, Cho CS and Weber SM: Staging laparoscopy enhances the detection of occult metastases in patients with pancreatic adenocarcinoma. J Surg Oncol 100(8): 663-669, 2009. PMID: 19780095. DOI: 10.1002/jso.21402
27 Morak MJ, Hermans JJ, Smeenk HG, Renders WM, Nuyttens JJ, Kazemier G and van Eijck CH: Staging for locally advanced pancreatic cancer. Eur J Surg Oncol 35(9): 963-968, 2009. PMID: 19780095. DOI: 10.1016/j.ejso.2009.01.013

28 Andriulli A, Festa V, Botteri E, Valvano MR, Koch M, Bassi C, Maisonneuve P and Sebastiano PD: Neoadjuvant/preoperative gemcitabine for patients with localized pancreatic cancer: A metaanalysis of prospective studies. Ann Surg Oncol 19(5): 1644-1662, 2012. PMID: 22012027. DOI: 10.1245/s10434-011-2110-8

29 Kim G: Nab-paclitaxel for the treatment of pancreatic cancer. Cancer Manag Res 9: 85-96, 2017. PMID: 28356771. DOI: 10.2147/CMAR.S127840

30 Yoshida K, Iwashita T, Uemura S, Maruta A, Okuno M, Ando N, Iwata K, Kawaguchi J, Mukai T and Shimizu M: A multicenter prospective phase ii study of first-line modified folfirinox for unresectable advanced pancreatic cancer. Oncotarget 8(67): 111346111355, 2017. PMID: 29340058. DOI: 10.18632/oncotarget.22795
Received June 24, 2019

Revised July 1, 2019

Accepted July 2, 2019 